\title{
Cognitive behavioral therapy for the management of poor sleep in insomnia disorder
}

\author{
This article was published in the following Dove Press journal: \\ ChronoPhysiology and Therapy \\ 17 November 2014 \\ Number of times this article has been viewed
}

\author{
Christopher B Miller ${ }^{1-3}$ \\ Colin A Espie ${ }^{4}$ \\ Simon D Kyle ${ }^{5}$ \\ 'NeuroSleep and Woolcock Institute \\ of Medical Research, University \\ of Sydney, Sydney, NSW, Australia; \\ ${ }^{2}$ Institute of Neuroscience and \\ Psychology, University of Glasgow, \\ Glasgow, UK; ${ }^{3}$ Sydney Nursing \\ School, The University of Sydney, \\ NSW, Australia; ${ }^{4}$ Sleep and Circadian \\ Neuroscience Institute (SCNi), \\ University of Oxford, Oxford, UK; \\ ${ }^{5}$ School of Psychological Sciences, \\ University of Manchester, Manchester, \\ UK
}

\begin{abstract}
Insomnia disorder is a common complaint of sleep disruption that impacts daytime performance and reduces quality of life. Insomnia is generally characterized by subjective reports of difficulty obtaining adequate sleep accompanied by daytime impairment. This review provides a brief overview of the classification, epidemiology, etiology, and models of the development of adult insomnia. The contemporary evidence base for cognitive behavioral therapy in insomnia is then reviewed, with a focus on sleep and daytime functioning. The review concludes with a discussion of emerging therapies, based on psychological/behavioral principles.
\end{abstract}

Keywords: insomnia, sleep, cognitive behavioral therapy, treatment, psychological intervention, review

\section{Classification of insomnia}

The American Psychiatric Association's Diagnostic and Statistical Manual of Mental Disorders, Fifth Edition (DSM-5) ${ }^{1}$ defines insomnia in adults as a complaint of dissatisfaction with sleep quantity or quality, associated with one (or more) of the following symptoms: 1) difficulty initiating sleep; 2) difficulty maintaining sleep characterized by frequent awakenings or problems returning to sleep after awakenings; or 3) early-morning awakening with inability to return to sleep. The sleep disturbance must also significantly impact on daytime functioning (social, occupational, educational, or academic) and occur at least three nights per week for at least three months. The impairment must also not be attributable to another disorder or explained by substance use. ${ }^{1}$ The DSM-5 no longer refers to "primary" and "secondary" insomnia, but considers insomnia to be a disorder in its own right when both nighttime and daytime criteria are met (regardless of comorbidity). ${ }^{1}$ Recently, the International Classification of Sleep Disorders, Third Edition (ICSD-3) has also moved toward defining insomnia under a more general and inclusive term of "insomnia disorder". ${ }^{2}$ Further refinements to the ICSD-3 criteria also include the removal of certain phenotypes of insomnia and an emphasis on daytime cognitive impairments (ie, working memory and attention; ${ }^{2,3}$ Table 1).

\section{Epidemiology}

Correspondence: Christopher B Miller Woolcock Institute of Medical Research, University of Sydney, PO Box M77, Missenden Road, Sydney, NSW 2050, Australia

Tel $+6|29| 14045 \mid$

Fax +6I 291140014

Email chris.miller@sydney.edu.au
Estimates of insomnia suggest that approximately one-third of adults report insomnia symptoms, making insomnia the most prevalent sleep disorder. ${ }^{1}$ Of those who suffer from insomnia, $10 \%$ will go on to develop chronic insomnia and will complain of insomnia in primary health care settings. These individuals also report daytime impairments due to poor sleep. ${ }^{4-6}$ 
Table I Criteria for insomnia disorder according to the Diagnostic and Statistical Manual of Mental Disorders, Fifth Edition' and the International Classification of Sleep Disorders, Third Edition ${ }^{2}$

\begin{tabular}{|c|c|c|}
\hline & DSM-5: insomnia disorder & ICSD-3: chronic insomnia disorder \\
\hline Sleep & $\begin{array}{l}\text { A predominant complaint of dissatisfaction with sleep quantity } \\
\text { or quality, associated with one (or more) of the following } \\
\text { symptoms: I) difficulty initiating sleep, } 2 \text { ) difficulty maintaining } \\
\text { sleep, characterized by frequent awakenings or problems } \\
\text { returning to sleep after awakenings, and } 3 \text { ) early-morning } \\
\text { awakening with inability to return to sleep. }\end{array}$ & $\begin{array}{l}\text { The patient reports (or the patient's parent or caregiver } \\
\text { reports) marked concern about, or dissatisfaction with, sleep } \\
\text { comprising one or more of the following: I) difficulty initiating } \\
\text { sleep, 2) difficulty maintaining sleep, 3) waking up earlier than } \\
\text { desired, 4) resistance in going to bed on the appropriate } \\
\text { schedule, and 5) difficulty sleeping without the parent } \\
\text { or caregiver present. }\end{array}$ \\
\hline Consequences & $\begin{array}{l}\text { The sleep disturbance causes clinically significant distress } \\
\text { or impairment in social, occupational, educational, academic, } \\
\text { behavioral, or other important areas of functioning. }\end{array}$ & $\begin{array}{l}\text { The patient reports (or the patient's parent or caregiver } \\
\text { reports) one or more of the following as being associated } \\
\text { with the nighttime sleep difficulty in fatigue; mood disturbance; } \\
\text { interpersonal problems; reduced cognitive function; reduced } \\
\text { performance; daytime sleepiness; behavioral problems } \\
\text { (eg, hyperactivity, impulsivity, aggression); reduced } \\
\text { motivation/initiative; proneness to errors/accidents. }\end{array}$ \\
\hline Frequency & The sleep difficulty occurs at least 3 nights per week. & $\begin{array}{l}\text { The sleep disturbance and associated daytime symptoms occur } \\
\text { at least } 3 \text { times per week. }\end{array}$ \\
\hline Chronicity & The sleep difficulty is present for at least 3 months. & $\begin{array}{l}\text { The sleep disturbance and associated daytime symptoms have } \\
\text { been present for at least } 3 \text { months. }\end{array}$ \\
\hline Exclusions & $\begin{array}{l}\text { The sleep difficulty occurs despite adequate opportunity for sleep. } \\
\text { The insomnia is not better explained by and does not occur } \\
\text { exclusively during the course of another sleep/wake disorder } \\
\text { (eg, narcolepsy, a breathing-related sleep disorder, a circadian } \\
\text { rhythm sleep/wake disorder, a parasomnia). } \\
\text { The insomnia is not attributable to the physiological effects } \\
\text { of a substance (eg, a drug of abuse, a medication). } \\
\text { Coexisting mental disorders and medical conditions do not } \\
\text { adequately explain the predominant complaint of insomnia. }\end{array}$ & $\begin{array}{l}\text { The reported sleep/wake complaints cannot be explained } \\
\text { purely by inadequate opportunity (ie, enough time is allotted } \\
\text { for sleep) or inadequate circumstances (ie, the environment } \\
\text { is safe, dark, quiet, and comfortable) for sleep. } \\
\text { The sleep/wake difficulty is not better explained by another } \\
\text { primary sleep disorder. }\end{array}$ \\
\hline
\end{tabular}

Abbreviations: DSM-5, Diagnostic and Statistical Manual of Mental Disorders, Fifth Edition; ICSD-3, International Classification of Sleep Disorders, Third Edition.

Prevalence varies widely depending upon criteria employed and whether it is considered a symptom of another disorder or an independent disorder. Frequently, insomnia is documented as a comorbid condition linked to another medical or psychiatric condition. For example, around half of individuals presenting with insomnia symptoms will also present with another psychiatric disorder. ${ }^{1}$ Insomnia is also more prevalent in females than males, with a ratio of about 1.44:1 and in those with lower social economic status. ${ }^{7}$ However, further epidemiological research is now required in order to elucidate the prevalence, natural history, and incidence of insomnia through time with longitudinal population-based cohort studies. This will enable researchers to understand if insomnia can be prevented and associated morbidity reduced. ${ }^{8}$ Insomnia is also a risk factor for comorbid medical and psychiatric disorders, including anxiety and depression. ${ }^{9-11}$

\section{Etiology and models of insomnia}

Insomnia is commonly conceptualized through a diathesis stress model referred to as the "3P model of insomnia". ${ }^{12}$ This model aims to provide a framework for the development and maintenance of chronic insomnia. ${ }^{13,14}$ The 3P model describes predisposing (behavior and genetic predisposition), precipitating (significant and often traumatic life events that may act as a trigger for insomnia), and perpetuating (behavior, mood, thoughts, and beliefs) factors that may contribute to both the development and maintenance of insomnia over time (Figure 1).

Spielman and Glovinsky ${ }^{15}$ suggest that certain individuals may be more vulnerable to the development of insomnia, depending upon predisposing factors like a heritable susceptibility to stress. Precipitating events can increase the likelihood of the development of insomnia in certain vulnerable or "at-risk" individuals and families. This may set off a period of poor sleep or acute short-term (1 month) insomnia. It is suggested that insomnia is maintained through perpetuating factors that reflect how an individual attempts to cope with sleep loss. For example, time in bed may be extended to increase the probability of achieving sleep. This, however, results in more time awake when in bed and more fragmented sleep due to a reduced homeostatic sleep drive. Time spent in bed may also vary day-to-day as the individual adjusts their sleep habits depending on the quality/quantity of sleep 


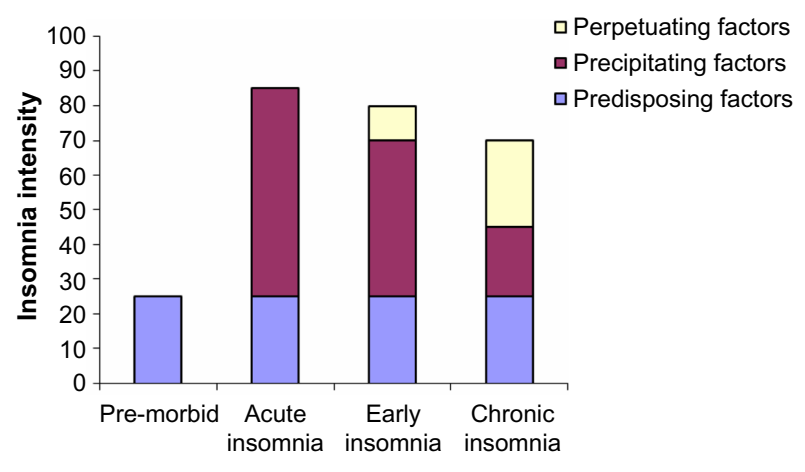

Figure I Factors contributing to the development of insomnia, according to the 3P framework.

Notes: Threshold refers to the probable intensity of insomnia to be classified as a "case". Reprinted from Elsevier Health Sciences, Espie CA, Kyle SD, Cognitive and behavioural psychological therapies for chronic insomnia, in: Therapy in Sleep Medicine, Barkoukis TJ, Matheson JK, Ferber R, Dohramji K (Ed.), I6I-17I, Copyright $\left(\right.$ ) 2012, with permission from Elsevier. ${ }^{80}$

previously achieved. This may then disrupt the circadian rhythm and also the predictability of sleep. ${ }^{12}$

In the development of insomnia, conditioned cues normally associated with sleep (eg, bed, bedroom, bedtime, increasing darkness) can become paired with wake activities or those that are not normally involved in sleep (eg, work or computer use). This results in faulty conditioning of maladaptive sleep habits, reinforcing symptoms of insomnia, preventing sleep onset, and possibly increasing sensitivity to further sources of disruption (noise, light, or temperature). Stimulus control therapy (SCT) and behavioral treatment for insomnia seeks to break these negative associations with the sleep environment. Attentional biases toward bedroom- and sleep-related stimuli have been described and are thought to provoke a conditioned response. ${ }^{16}$ Cognitive models of insomnia also posit that insomnia is maintained by dysfunctional cognitive processes, including excessive worry, anxiety, and catastrophizing, which negatively impact on sleep. Indeed, Harvey ${ }^{17}$ suggests that in line with anxiety literature, attention needs to be placed on daytime factors in insomnia that equally disrupt nighttime sleep. Those with insomnia may overestimate daytime performance deficits and this may cause increased anxiety over sleep loss and in turn reinforce worry regarding obtaining enough sleep. ${ }^{17}$

Both cognitive and neurocognitive models of insomnia build on the initial behavioral perspective and recognize that insomnia is precipitated by stressful life events. ${ }^{18}$ Neurocognitive models argue that increased cortical arousal (measured through cerebral electroencephalographic rhythms) cause increased sensory/information processing and disrupt sleep initiation and maintenance. ${ }^{18}$ This increased sensory/ information processing appears to contribute to an attenuated mesograde amnesia of sleep in insomnia or reduced forgetting of sleep. ${ }^{18,19}$ Sleep is therefore difficult to accurately quantify in insomnia; however, a number of different measures can be used including sleep diaries, actigraphy, and overnight sleep studies. ${ }^{20}$ Once conditioned arousal has set in, it potentially perpetuates long-term insomnia through preoccupation of sleep. This has previously been proposed as part of an attention-intention-effort pathway, which highlights that when increased effort is placed on attempting sleep, this in turn may cause further sleep loss due to the disruption of the automaticity of sleep. ${ }^{16,21}$ Cognitive behavioral therapy for insomnia (CBT-I) looks to address this increased sleep effort by modifying thoughts, beliefs, and behaviors of sleep. ${ }^{22}$

\section{Treatment of insomnia}

Evidence-based treatment and management for adult insomnia can be distilled into two mainstay modalities: 1) pharmacotherapy or 2) CBT-I. Prior to seeking treatment directly from a health care professional (many with insomnia do not seek treatment), individuals often report attempting alcohol, over-the-counter medications (eg, antihistamines), or herbal/dietary interventions to alleviate symptoms..$^{23,24}$ Non-evidence-based treatments for insomnia, however, do have the potential to further disrupt sleep and may cause harm and/or dependence (eg, alcohol, marijuana).

\section{Pharmacotherapy}

In primary care settings, insomnia is generally treated with prescription medications such as benzodiazepines (eg, temazepam, flurazepam). Despite a recent fall in prescribing benzodiazepines, overall hypnotic prescription rates have increased due to a rise in non-benzodiazepine receptor agonists or "z-drugs" (eg, zolpidem, zaleplon, eszopiclone). ${ }^{25}$ Z-drugs are thought to be more effective due to functional selectivity for specific receptor subunits and are also safer with a shorter half-life than benzodiazepines. However, there is a lack of evidence for less adverse effects, potential for tolerance, or long-term effectiveness ( $>4$ weeks). ${ }^{26,27}$ Sedative aids such as antihistamines (doxylamine) and melatonin receptor agonists can also be used to treat insomnia. Melatonin has been approved in those over 55 years in the UK for short-term use ( $<4$ weeks) but evidence is lacking regarding the effectiveness of melatonin in the long term. ${ }^{28} \mathrm{~A}$ number of off-label agents are often used to treat insomnia despite a paucity of evidence, including antidepressants (eg, trazodone, trimipramine, doxepin), antipsychotics (eg, quetiapine), and anticonvulsants (eg, gabapentin) ${ }^{28}$ Newer pharmacotherapy strategies now look to target the hypocretin/orexin receptor 
antagonist with suvorexant, which appears beneficial in the short term (first 4 weeks). ${ }^{29,30}$

\section{CBT-I}

The main nonpharmacological treatment for insomnia is CBT-I, which is an evidence-based psychological intervention, usually delivered by a psychologist individually, in small groups, or through automated web-based programs. ${ }^{31,32}$ CBT-I seeks to manage insomnia by targeting maladaptive thoughts, behaviors, and beliefs about sleep. ${ }^{33}$ CBT-I is a multicomponent approach and in the context of clinical trials improves sleep in $70 \%$ of insomnia patients. ${ }^{9,34}$ CBT-I has been found to have similar benefits to pharmacotherapy in the short term but with lasting treatment effects over pharmacotherapy, ${ }^{27,35}$ and is considered the first-line treatment for insomnia by the American Academy of Sleep Medicine, ${ }^{24}$ National Institutes of Health, ${ }^{36}$ and British Association of Psychopharmacology. ${ }^{37}$ Furthermore, the effectiveness of CBT-I is not moderated by factors including: age, sex, hypnotics, or comorbidity. ${ }^{24,38}$ The overall approach consists of five main treatment strategies (Table 2).

CBT-I now has meta-analytical evidence to suggest robust improvements in subjective measures of sleep (Figure 2). Sleep diaries have been found to show medium-to-large effect size (ES) improvements (range $d=0.77-1.13$ pre-to-post treatment) for outcome measures, including sleep onset latency (SOL), wake time after sleep onset (WASO), and sleep efficiency ( $\% \mathrm{SE}$ : the ratio of time spent in bed asleep).
Total sleep time (TST) was also found to improve with a small $\operatorname{ES}(d=0.29) .{ }^{39}$ In a meta-analysis, Ho et $\mathrm{al}^{40}$ recently found that self-help CBT-I improved sleep, anxiety, and depressive symptoms with medium-to-large ES scores for $\% \mathrm{SE}(d=0.80)$, $\operatorname{SOL}(d=0.66)$, and WASO $(d=0.55)$. Interestingly the dropout rate for self-help CBT-I was comparable to therapist-delivered CBT-I (14.5\% versus $16.7 \%$, respectively). ${ }^{40}$ Similar results have been documented previously as Irwin et $\mathrm{al}^{41}$ and Okajima et $\mathrm{al}^{42}$ also found medium-to-large ES improvements for SOL ( $d=0.52$ and $d=0.67$, respectively) and \% SE $(d=0.52$ and $d=0.89$, respectively) and medium-to-large ES reductions for WASO ( $d=0.64$ and $d=0.70)$. Small-to-medium ES increases were also found across meta-analytic studies for TST (range $d=0.17-0.46){ }^{35,41,42}$ Objectively defined sleep variables (polysomnography and actigraphy) have also shown small-to-moderate effects after CBT-I in metaanalytical studies, including SOL $(d=0.58)$, WASO $(d=0.57)$, and $\% \mathrm{SE}(d=0.47){ }^{42}$ however, measures of objective sleep are generally lacking in studies. Further studies adequately quantifying objective measures before, after, and at follow-up (12 months) are now required as part of randomized controlled trials (RCTs) to evaluate whether objective outcomes of therapy are sustained long term.

\section{Daytime functioning}

Given that daytime dysfunction is attributed to poor sleep in insomnia, it would seem important for trials to examine both nighttime and daytime outcomes pre-to-post CBT-I. However,

Table 2 Psychological and behavioral therapies for insomnia

Treatment (level of endorsement)

Stimulus control therapy (standard)

Behavioural recommendations designed to reinforce the association between the bed or bedroom and sleep, and to strengthen a consistent sleepwake schedule: (a) go to bed only when sleepy; (b) get out of bed when unable to sleep; (c) use the bed for sleep only (no reading, problem-solving in bed); (d) arise at the same time every morning; (e) avoid napping.

Sleep restriction therapy (guideline)

A method that limits the time spent in bed as close as possible to the actual sleep time, thereby producing a mild sleep deprivation, which results in more consolidated sleep. The sleep window is gradually increased throughout a few days or weeks until optimum sleep duration is achieved.

Relaxation training (standard)

Clinical procedures aimed at reduction of somatic tension (eg, progressive muscle relaxation, autogenic training) or intrusive thoughts (eg, imagery training, meditation) interfering with sleep. Most relaxation techniques need professional guidance initially and daily practice for a few weeks.

Cognitive therapy (insufficient evidence as single therapy)

Psychotherapeutic method aimed at alleviating excessive worries and revising misconceptions about sleep, insomnia, and daytime consequences. Specific targets include unrealistic sleep expectations, fear of the consequences of insomnia, and misconceptions of the causes of insomnia.

Sleep hygiene education (insufficient evidence as single therapy)

General guidelines about health practices (eg, diet, exercise, substance use) and environmental factors (eg, light, noise, temperature) that might promote or interfere with sleep: (a) avoid stimulants (eg, caffeine, nicotine) for several hours before bedtime; (b) avoid alcohol around bedtime as it fragments sleep during the second half of the night; (c) exercise regularly, it can deepen sleep; (d) do not watch the clock; (e) keep the bedroom environment dark, quiet, and comfortable.

Cognitive behavioural therapy (standard)

A combination of any of the above behavioural (eg, sleep restriction, stimulus control instructions, relaxation) and cognitive procedures. Note: Reprinted from The Lancet, 379(982I), Morin CM, Benca R, Chronic insomnia. Lancet, II29-II4I, Copyright @ 20I2, with permission from Elsevier.9 


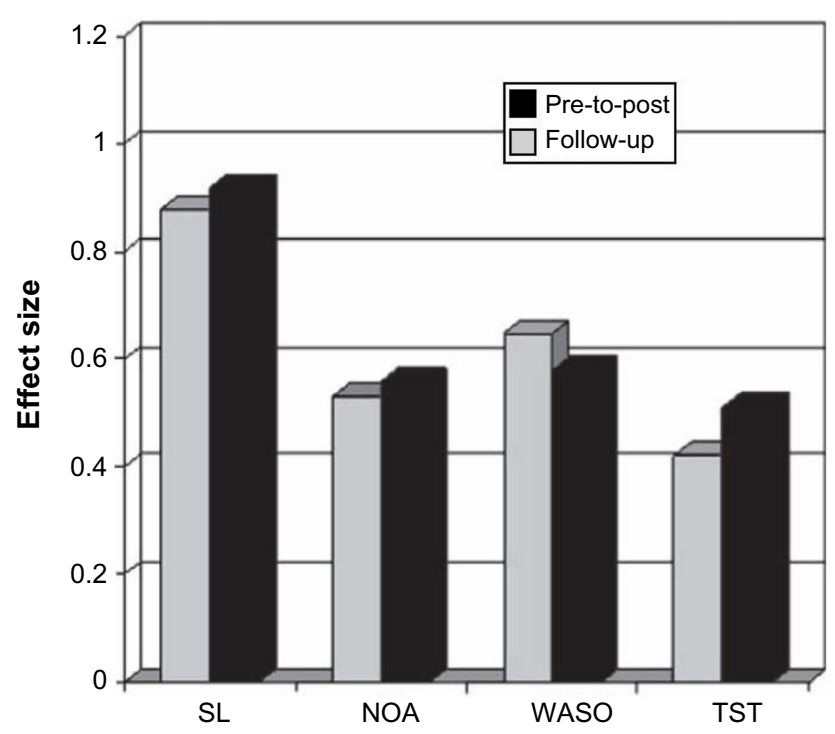

Figure 2 Effect sizes for psychological and behavioral interventions pre-to-post treatment and at follow-up (average of 6 months) for cognitive behavioral therapy for insomnia

Note: Reprinted from Sleep Medicine Reviews, Vol I3, Issue 3, Riemann D, Perlis ML, The treatments of chronic insomnia: A review of benzodiazepine receptor agonists and psychological and behavioral therapies, Pages 205-214. ${ }^{27}$ Copyright (C) 2008, with permission from Elsevier.

Abbreviations: NOA, number of awakenings; SL, sleep latency; TST, total sleep time; WASO, wake time after sleep onset.

in general, CBT-I treatment studies have rarely assessed and/or reported daytime functioning outcomes. Indeed, since the last recommendations for a standard research assessment of insomnia formulated by 25 experts and published in 2006 , no CBT-I treatment study has reported all of the recommended "essential" measures suggested by this group. ${ }^{43,44}$ Therefore, more studies are now required to not only evaluate sleep but also measures of daytime functioning in response to CBT-I. Generally, in the studies that have attempted to measure daytime functioning, improvements have been found for subjective measures of anxiety, depression, fatigue, and quality of life. ${ }^{45-49}$ However, a number of studies have also failed to find any clinically significant treatment improvements. This may be due to the use of generic measures (eg, the Short Form-36 Health Survey: a measure of health related quality of life) that do not sufficiently take into account the specific domains of functioning relevant to the individual ${ }^{50}$ and/or because the patients sampled are relatively healthy, making it difficult to document a significant effect of treatment. ${ }^{44}$

Treatment studies should now look to also measure subjective daytime functioning in those with insomnia and in response to CBT-I. This is due to the fact that insomnia requires an explicit impairment of daytime functioning for it to be considered a disorder ${ }^{1,2}$ and a true 24-hour problem that impacts on both sleep and wake. Daytime impairments can include global complaints of functioning in areas of social, occupational, or relationship functioning or can also present as a more specific complaint in areas of mood, concentration, impaired memory performance, or fatigue for example. ${ }^{1,2,51-53}$ It is also thought that these daytime impairments contribute to reduced ratings of overall health-related quality of life ${ }^{49}$ and drive treatment-seeking behavior. ${ }^{54}$ In addition to subjective reports, objective assessments of neurocognition and emotion perception may also be considered as dependent variables in light of documented impairments. ${ }^{3,55,56}$

\section{Treatment delivery of CBT-I}

The primary problem with CBT-I is accessing treatment and this is due to a shortage of trained health care professionals who can deliver CBT-I. CBT-I can be delivered in a number of different procedures and it is this aspect of the literature that has received recent attention. Historically, a clinical psychologist trained specifically in CBT-I would deliver treatment. However, the volume of patients seeking treatment for insomnia and the limited number of trained psychologists means that access to therapy is difficult. ${ }^{31}$ Stepped care is potentially an extremely useful and cost effective framework for the dissemination of CBT-I. This strategy aims to treat the majority of patients with minimal entry level treatments that are less costly and easier to deliver than full CBT-I (eg, Internet delivered, ${ }^{57}$ self-help booklets, ${ }^{58}$ brief therapies; ${ }^{59}$ Table 3). Nonresponders who present with more complex problems are able to refer up to higher levels of treatment that are more resource intensive and delivered by a specialist trained in behavioral sleep medicine. ${ }^{31,32}$

Strategies to deliver a stepped care service effectively in primary care based-settings taking account of both time and personnel factors are now required. To address this, studies have attempted to manualize therapy with nurse practitioners ${ }^{47,60}$ in order to provide brief primary care "friendly" versions of CBT-I. ${ }^{59}$ Buysse et al ${ }^{61}$ found that the delivery of sleep restriction therapy (SRT) plus SCT for insomnia over two sessions and two follow-up telephone calls produced significantly better

Table 3 Treatment modalities of cognitive behavioral therapy for insomnia and key controlled trials

\begin{tabular}{ll}
\hline Group therapy & Espie et al ${ }^{47,76,77}$ \\
Large group therapy & Jernelov et $\mathrm{al}^{78}$ \\
Individual therapy & Morin et al ${ }^{34}$ \\
Brief therapy & Edinger and Sampson ${ }^{59}$ \\
Telephone-delivered & Arnedt et al $^{79}$ \\
Internet-delivered & Espie et al ${ }^{57}$ \\
Self-help books & Jernelov et al $^{78}$ \\
Self-help booklets & Morgan et al ${ }^{58}$ \\
\hline
\end{tabular}


outcomes in sleep diary and actigraphic measures of sleep compared to a sleep information control condition in older adults with insomnia. Gains were also maintained at 6 months follow-up. More recently, studies have looked to evaluate the individual contributions of behavioral and cognitive-based approaches of overall CBT-I. ${ }^{62}$

Stand-alone behavioral components of CBT-I have been evaluated recently as part of a dismantling approach to insomnia treatment. As part of an RCT, Epstein et $\mathrm{al}^{63}$ assessed the contribution of multicomponent therapy (consisting of SRT plus SCT with no structured cognitive approach) compared to SRT, SCT, and waitlist control groups. The study found SCT plus SRT to be as effective as multicomponent therapy and suggests that individual behavioral treatment options may be powerful stand-alone interventions. ${ }^{64,65}$ Indeed, such components may be delivered more rapidly than overall CBT-I in primary care settings by trained nurse practitioners or Masters level therapists as part of a stepped care framework. ${ }^{31}$ Therefore, access to overall CBT-I is perhaps essential as patients may respond individually to different components of CBT-I. A stepped care approach can provide access to patients who do not respond initially to low cost or manualized interventions. The self-help strategies facilitated by treatment modalities like the Internet may now be considered as the first port of call into the overall referral pathway of stepped care for insomnia. ${ }^{32,40}$ Recently, RCTs have evaluated the use of the Internet to deliver a standardized and low-cost form of CBT-I and found improvements in subjective measures of both sleep and daytime functioning. ${ }^{57,66}$ The integration of this approach into primary care is a current area of interest that should be adopted and evaluated for treatment efficacy; this aims to improve access to CBT-I and enable treatment to be the first approach to insomnia management.

\section{Emerging therapies}

Recently, new psychological treatments for insomnia have been proposed with preliminary evidence from clinical trials. The first is called intensive sleep retraining (ISR) and involves an in-laboratory protocol to recondition the automatic process of sleep initiation through multiple sleep onset trials. Retraining consists of 50 half-hourly sleep onset trials over a 25-hour sleep deprivation period. Each opportunity for sleep onset is limited to 20 minutes and stopped if sleep does not occur. If sleep does occur, it is limited to three consecutive minutes only and then the patient is awoken. ${ }^{67,68}$ The procedure is relatively novel and so far only one RCT has evaluated the efficacy of ISR. Treatment appeared to rapidly improve SOL and TST in those who were in the retraining condition combined with a behavioral intervention (SCT). Therefore, the findings have suggested that this in-laboratory protocol may quickly recondition the patient with insomnia to sleeppromoting stimuli (bed, bedroom environment, and factors involved in sleep initiation). ISR may be a powerful and relatively fast way to administer what is normally achieved over a number of weeks with SRT and SCT procedures addressing the wake-promoting activities that have been paired with sleep onset. ${ }^{69}$ This intervention may also have therapeutic effects on cognitive processes associated with insomnia maintenance, including dysfunctional thoughts and beliefs about sleep and sleep effort. One limitation of this approach is the implementation of the experimental procedure, which is costly due to the amount of time required in the laboratory. Therefore, ISR is in its infancy and further studies are required to examine the clinical effectiveness and provide a suitable method for patient delivery (eg, homebased procedure).

Further research has looked at the contribution of combining pharmacotherapy with CBT-I in order to increase adherence and the potentiation of treatment outcomes systematically. Morin et $\mathrm{al}^{34}$ evaluated the use of CBT-I combined with zolpidem compared to CBT-I alone and found enhanced benefits during the acute phase of therapy (first 6 weeks) only. Long-term treatment was most effective with discontinuation of medication. One other previous study, however, found CBT-I to be more effective compared to combined therapy (CBT-I with zolpidem). ${ }^{70}$ Therefore, CBT-I should be considered the first-line intervention for insomnia, and hypnotics may only produce short-term gains above CBT-I. These results reinforce previous findings regarding the short-term only use of pharmacotherapy for insomnia. ${ }^{27,35,71,72}$

One further development in the literature is to specifically target cognitive processes (worry and rumination; attentional bias/sleep-related monitoring; misperception of sleep and daytime deficits; unhelpful beliefs about sleep; use of safety behaviors) that operate both during the day and night to maintain insomnia. A preliminary open trial of cognitive therapy (CT) for insomnia found improvements in both nighttime and daytime factors, which were found to be maintained at 12 months follow-up, suggesting that this may be a useful therapy for insomnia. ${ }^{73}$ Harvey et $\mathrm{al}^{62}$ next looked to evaluate the use of CT as part of an RCT for the treatment of chronic insomnia with the aim of evaluating the individual contributions of behavior therapy and CT compared to overall CBT-I for insomnia. This study found that full CBT-I had the greatest improvements (proportion of treatment responders quantified by the insomnia severity index). Behavior therapy 
was found to have the fastest improvements but these were not maintained at 6 months follow-up, whereas gains with CT were the slowest but were found to be similar to CBT-I at follow-up. Therefore, this study suggests that $\mathrm{CT}$ is required for maintaining improvements in sleep. ${ }^{62}$ Further RCTs are now required to evaluate the specific treatment effectiveness of CT and newer "third-wave" treatment approaches, including mindfulness. ${ }^{74,75}$

\section{Conclusion}

Insomnia is a common sleep disorder that impacts on both sleep and daytime functioning. CBT-I is an effective intervention for the treatment of insomnia for sleep diary variables but more work is required and attention should be placed on further measures of subjective daytime functioning and of objective sleep changes. Further research is required to systematically examine the clinical effectiveness of emerging therapies for insomnia through well-designed, large-scale RCTs.

\section{Acknowledgments}

This research was supported by the National Health and Medical Research Council (NHMRC; Australia), NeuroSleep (grant APP1060992).

\section{Disclosure}

Professor Colin Espie has conducted research for and is Clinical and Scientific Director of Sleepio Limited. He has consulted for Boots UK and Novartis, has participated in speaking engagements for UCB and Novartis; and has used actigraphs supplied at no charge by Philips Respironics in research studies. Dr Kyle has conducted research for Sleepio Limited. The authors report no other conflicts of interest in this work.

\section{References}

1. American Psychiatric Association. Diagnostic and Statistical Manual of Mental Disorders. 5th ed. Arlington, VA, USA: American Psychiatric Publishing; 2013.

2. American Academy of Sleep Medicine. International Classification of Sleep Disorders. 3rd ed. Darien, IL, USA: American Academy of Sleep Medicine; 2014.

3. Fortier-Brochu E, Beaulieu-Bonneau S, Ivers H, Morin CM. Insomnia and daytime cognitive performance: a meta-analysis. Sleep Med Rev. 2012;16(1):83-94.

4. Bartlett DJ, Marshall NS, Williams A, Grunstein RR. Sleep health New South Wales: chronic sleep restriction and daytime sleepiness. Intern Med J. 2008;38(1):24-31.

5. Morphy H, Dunn KM, Lewis M, Boardman HF, Croft PR. Epidemiology of insomnia: a longitudinal study in a UK population. Sleep. 2007;30(3):274-280.

6. Ohayon MM, Hong SC. Prevalence of insomnia and associated factors in South Korea. J Psychosom Res. 2002;53(1):593-600.
7. Pallesen S, Sivertsen B, Nordhus IH, Bjorvatn B. A 10-year trend of insomnia prevalence in the adult Norwegian population. Sleep Med. 2014;15(2):173-179.

8. Morin CM, Jarrin DC. Epidemiology of insomnia: prevalence, course, risk factors, and public health burden. Sleep Med Clin. 2013;8(3): 281-297.

9. Morin CM, Benca R. Chronic insomnia. Lancet. 2012;379(9821): 1129-1141.

10. Sivertsen B, Salo P, Mykletun A, et al. The bidirectional association between depression and insomnia: the HUNT study. Psychosom Med. 2012;74(7):758-765.

11. Budhiraja R, Roth T, Hudgel DW, Budhiraja P, Drake CL. Prevalence and polysomnographic correlates of insomnia comorbid with medical disorders. Sleep. 2011;34(7):859-867.

12. Spielman AJ, Yang CM, Glovinsky PB. Insomnia: sleep restriction therapy. In: Sateia MJ, Buysse DJ, editors. Insomnia: Diagnosis and Treatment. London: Informa Healthcare; 2010: 277-289.

13. Buysse DJ, Germain A, Hall M, Monk TH, Nofzinger EA. A neurobiological model of insomnia. Drug Discov Today Dis Models. 2011;8(4):129-137.

14. Glovinsky PB, Spielman AJ. Sleep restriction therapy. In: Hauri PJ, editor. Case Studies in Insomnia. New York, NY: Kluwer Academic; 1991:49-63.

15. Spielman AJ, Glovinsky PB. The varied nature of insomnia. In: Hauri JP, editor. Case Studies in Insomnia. New York, NY: Kluwer Academic; 1991:1-15.

16. Espie CA, Broomfield NM, MacMahon KM, Macphee LM, Taylor LM. The attention-intention-effort pathway in the development of psychophysiologic insomnia: a theoretical review. Sleep Med Rev. 2006; 10(4):215-245.

17. Harvey AG. A cognitive model of insomnia. Behav Res Ther. 2002;40(8):869-893.

18. Perlis ML, Giles DE, Mendelson WB, Bootzin RR, Wyatt JK. Psychophysiological insomnia: the behavioural model and a neurocognitive perspective. J Sleep Res. 1997;6(3):179-188.

19. Perlis ML, Smith MT, Andrews PJ, Orff H, Giles DE. Beta/gamma EEG activity in patients with primary and secondary insomnia and good sleeper controls. Sleep. 2001;24(1):110-117.

20. Miller CB, Kyle SD, Melehan KL, Bartlett DJ. Methodology for the assessment of sleep. In: Babson KA, Feldner MT, editors. Sleep and Affect: Assessment, Theory, and Clinical Implications. San Diego, CA: Elsevier Academic Press; 2014.

21. Rasskazova E, Zavalko I, Tkhostov A, Dorohov V. High intention to fall asleep causes sleep fragmentation. $J$ Sleep Res. 2014;23(3): 295-301.

22. Perlis M, Shaw PJ, Cano G, Espie CA. Models of insomnia. In: Kryger MH, Roth T, Dement WC, editors. Principles and Practice of Sleep Medicine. 5th ed. St Louis: Elsevier Saunders; 2011:850-865.

23. Krystal AD. Treating the health, quality of life, and functional impairments in insomnia. J Clin Sleep Med. 2007;3(1):63-72.

24. Morin CM, Bootzin RR, Buysse DJ, Edinger JD, Espie CA, Lichstein KL. Psychological and behavioral treatment of insomnia: update of the recent evidence (1998-2004). Sleep. 2006;29(11):1398-1414.

25. Huedo-MedinaTB, Kirsch I, Middlemass J, Klonizakis M, SiriwardenaAN. Effectiveness of non-benzodiazepine hypnotics in treatment of adult insomnia: meta-analysis of data submitted to the Food and Drug Administration. BMJ. 2012;345:e8343.

26. Nutt DJ, Stahl SM. Searching for perfect sleep: the continuing evolution of GABAA receptor modulators as hypnotics. J Psychopharmacol. 2010;24(11):1601-1612.

27. Riemann D, Perlis ML. The treatments of chronic insomnia: a review of benzodiazepine receptor agonists and psychological and behavioral therapies. Sleep Med Rev. 2009;13(3):205-214.

28. Minkel J, Krystal AD. Optimizing the pharmacologic treatment of insomnia: current status and future horizons. Sleep Med Clin. 2013;8(3):333-350. 
29. Michelson D, Snyder E, Paradis E, et al. Safety and efficacy of suvorexant during 1-year treatment of insomnia with subsequent abrupt treatment discontinuation: a Phase III randomised, double-blind, placebo-controlled trial. Lancet Neurol. 2014;13(5):461-471.

30. Herring WJ, Snyder E, Budd K, et al. Orexin receptor antagonism for treatment of insomnia: a randomized clinical trial of suvorexant. Neurology. 2012;79(23):2265-2274.

31. Espie CA. "Stepped care": a health technology solution for delivering cognitive behavioral therapy as a first line insomnia treatment. Sleep. 2009;32(12):1549-1558.

32. Espie CA, Hames P, McKinstry B. Use of the Internet and mobile media for delivery of cognitive behavioral insomnia therapy. Sleep Med Clin. 2013;8(3):407-419.

33. Edinger JD, Means MK. Cognitive-behavioral therapy for primary insomnia. Clin Psychol Rev. 2005;25(5):539-558.

34. Morin CM, Vallieres A, Guay B, et al. Cognitive behavioral therapy, singly and combined with medication, for persistent insomnia a randomized controlled trial. JAMA. 2009;301(19):2005-2015.

35. Smith MT, Perlis ML, Park A, et al. Comparative meta-analysis of pharmacotherapy and behavior therapy for persistent insomnia. Am J Psychiatry. 2002;159(1):5-11.

36. National Institutes of Health State-of-the-Science Conference Statement on manifestations and management of chronic insomnia in adults. $\mathrm{NIH}$ Consens State Sci Statements. 2005;22(2):1-30.

37. Wilson SJ, Nutt DJ, Alford C, et al. British Association for Psychopharmacology consensus statement on evidence-based treatment of insomnia, parasomnias, and circadian rhythm disorders. J Psychopharmacol. 2010;24(11):1577-1601.

38. Espie CA, Inglis SJ, Harvey L. Predicting clinically significant response to cognitive behavior therapy for chronic insomnia in general medical practice: analysis of outcome data at 12 months posttreatment. $J$ Consult Clin Psych. 2001;69(1):58-66.

39. Koffel EA, Koffel JB, Gehrman PR. A meta-analysis of group cognitive behavioral therapy for insomnia. Sleep Med Rev. Epub May 14, 2014.

40. Ho FY, Chung KF, Yeung WF, et al. Self-help cognitive-behavioral therapy for insomnia: a meta-analysis of randomized controlled trials. Sleep Med Rev. Epub July 9, 2014.

41. Irwin MR, Cole JC, Nicassio PM. Comparative meta-analysis of behavioral interventions for insomnia and their efficacy in middle-aged adults and in older adults 55+ years of age. Health Psychol. 2006;25(1):3-14.

42. Okajima I, Komada Y, Inoue Y. A meta-analysis on the treatment effectiveness of cognitive behavioral therapy for primary insomnia. Sleep Biol Rhythms. 2011;9(1):24-34.

43. Buysse DJ, Ancoli-Israel S, Edinger JD, Lichstein KL, Morin CM Recommendations for a standard research assessment of insomnia. Sleep. 2006;29(9):1155-1173.

44. Taylor DJ, Zimmerman MR, Gardner CE, et al. A pilot randomized controlled trial of the effects of cognitive-behavioral therapy for insomnia on sleep and daytime functioning in college students. Behav Ther. 2014;45(3):376-389.

45. Belleville G, Morin CM. Hypnotic discontinuation in chronic insomnia: impact of psychological distress, readiness to change, and self-efficacy. Health Psychol. 2008;27(2):239-248.

46. Dirksen SR, Epstein DR. Efficacy of an insomnia intervention on fatigue, mood, and quality of life in breast cancer survivors. JAdv Nurs. 2008;61(6):664-675.

47. Espie CA, MacMahon KM, Kelly HL, et al. Randomized clinical effectiveness trial of nurse-administered small-group cognitive behavior therapy for persistent insomnia in general practice. Sleep. 2007;30(5):574-584.

48. Quesnel C, Savard J, Simard S, Ivers H, Morin CM. Efficacy of cognitive-behavioral therapy for insomnia in women treated for nonmetastic breast cancer. J Consult Clin Psych. 2003;71(1):189-200.

49. Kyle SD, Morgan K, Espie CA. Insomnia and health-related quality of life. Sleep Med Rev. 2010;14(1):69-82.
50. Kyle SD, Crawford MR, Morgan K, Spiegelhalder K, ClarkAA, Espie CA. The Glasgow Sleep Impact Index (GSII): a novel patient-centred measure for assessing sleep-related quality of life impairment in insomnia disorder. Sleep Med. 2013;14(6):493-501.

51. Kyle SD, Espie CA, Morgan K. “... Not just a minor thing, it is something major, which stops you from functioning daily": quality of life and daytime functioning in insomnia. Behav Sleep Med. 2010;8(3):123-140.

52. Edinger JD, Bonnet MH, Bootzin RR, et al. Derivation of research diagnostic criteria for insomnia: report of an American Academy of Sleep Medicine Work Group. Sleep. 2004;27(8):1567-1596.

53. Espie CA, Kyle SD, Hames P, Cyhlarova E, Benzeval M. The daytime impact of DSM-5 insomnia disorder: comparative analysis of insomnia subtypes from the Great British Sleep Survey. J Clin Psychiatry. 2012;73(12):e1478-e1484.

54. Morin C, LeBlanc M, Daley M, Gregoire JP, Merette C. Epidemiology of insomnia: prevalence, self-help treatments, consultations, and determinants of help-seeking behaviors. Sleep Med. 2006;7(2): 123-130.

55. Kyle SD, Beattie L, Spiegelhalder K, Rogers Z, Espie CA. Altered emotion perception in insomnia disorder. Sleep. 2014;37(4):775-783.

56. Altena E, Van Der Werf YD, Strijers RL, Van Someren EJ. Sleep loss affects vigilance: effects of chronic insomnia and sleep therapy. J Sleep Res. 2008;17(3):335-343.

57. Espie CA, Kyle SD, Williams C, et al. A randomized, placebo-controlled trial of online cognitive behavioral therapy for chronic insomnia disorder delivered via an automated media-rich web application. Sleep. 2012;35(6):769-781.

58. Morgan K, Gregory P, Tomeny M, David BM, Gascoigne C. Selfhelp treatment for insomnia symptoms associated with chronic conditions in older adults: a randomized controlled trial. J Am Geriatr Soc. 2012;60(10):1803-1810.

59. Edinger JD, Sampson WS. A primary care "friendly" cognitive behavioral insomnia therapy. Sleep. 2003;26(2):177-184.

60. Bothelius K, Kyhle K, Espie CA, Broman JE. Manual-guided cognitivebehavioural therapy for insomnia delivered by ordinary primary care personnel in general medical practice: a randomized controlled effectiveness trial. J Sleep Res. 2013;22(6),688-696.

61. Buysse DJ, Germain A, Moul DE, et al. Efficacy of brief behavioral treatment for chronic insomnia in older adults. Arch Intern Med. 2011;171(10):887-895.

62. Harvey AG, Belanger L, Talbot L, et al. Comparative efficacy of behavior therapy, cognitive therapy, and cognitive behavior therapy for chronic insomnia: a randomized controlled trial. J Consult Clin Psych. 2014;82(4):670-683.

63. Epstein DR, Sidani S, Bootzin RR, Belyea MJ. Dismantling multicomponent behavioral treatment for insomnia in older adults: a randomized controlled trial. Sleep. 2012;35(6):797-805.

64. Miller CB, Kyle SD, Marshall NS, Espie CA. Ecological momentary assessment of daytime symptoms during sleep restriction therapy for insomnia. J Sleep Res. 2013;22(3):266-272.

65. Miller CB, Espie CA, Epstein DR, et al. The evidence base of sleep restriction therapy for treating insomnia disorder. Sleep Med Rev. 2014;18(5):415-424

66. Ritterband LM, Thorndike FP, Gonder-Frederick LA, et al. Efficacy of an Internet-based behavioral intervention for adults with insomnia. Arch Gen Psychiatry. 2009;66(7):692-698.

67. Harris J, Lack L, Kemp K, Wright H, Bootzin R. A randomized controlled trial of intensive sleep retraining (ISR): a brief conditioning treatment for chronic insomnia. Sleep. 2012;35(1):49-60.

68. Harris J, Lack L, Wright H, Gradisar M, Brooks A. Intensive sleep retraining treatment for chronic primary insomnia: a preliminary investigation. J Sleep Res. 2007;16(3):276-284.

69. Bootzin RR. Stimulus control treatment for insomnia. In: Proceedings of the 80th Annual Convention of the American Psychological Association. Washington, DC: American Psychological Association; 1972: 395-396. 
70. Jacobs GD, Pace-Schott EF, Stickgold R, Otto MW. Cognitive behavior therapy and pharmacotherapy for insomnia: a randomized controlled trial and direct comparison. Arch Intern Med. 2004;164(17):1888-1896.

71. Morin CM, Colecchi C, Stone J, Sood R, Brink D. Behavioral and pharmacological therapies for late-life insomnia: a randomized controlled trial. JAMA. 1999;281(11):991-999.

72. Morin CM, Beaulieu-Bonneau S, Ivers H, et al. Speed and trajectory of changes of insomnia symptoms during acute treatment with cognitivebehavioral therapy, singly and combined with medication. Sleep Med 2014;15(6):701-707.

73. Harvey AG, Sharpley AL, Ree MJ, Stinson K, Clark DM. An open trial of cognitive therapy for chronic insomnia. Behav Res Ther. 2007;45(10): 2491-2501.

74. Ong JC, Shapiro SL, Manber R. Mindfulness meditation and cognitive behavioral therapy for insomnia: a naturalistic 12-month follow-up. Explore (NY). 2009;5(1):30-36.

75. Ong JC, Shapiro SL, Manber R. Combining mindfulness meditation with cognitive-behavior therapy for insomnia: a treatment-development study. Behav Ther. 2008;39(2):171-182.
76. Espie CA, Inglis SJ, Tessier S, Harvey L. The clinical effectiveness of cognitive behaviour therapy for chronic insomnia: implementation and evaluation of a sleep clinic in general medical practice. Behav Res Ther. 2001;39(1):45-60.

77. Espie CA, Fleming L, Cassidy J, et al. Randomized controlled clinical effectiveness trial of cognitive behavior therapy compared with treatment as usual for persistent insomnia in patients with cancer. J Clin Oncol. 2008;26(28):4651-4658.

78. Jernelov S, Lekander M, Blom K, et al. Efficacy of a behavioral selfhelp treatment with or without therapist guidance for co-morbid and primary insomnia: a randomized controlled trial. BMC Psychiatry. 2012;12(1):5.

79. Arnedt JT, Cuddihy L, Swanson LM, Pickett S, Aikens J, Chervin RD. Randomized controlled trial of telephone-delivered cognitive behavioral therapy for chronic insomnia. Sleep. 2013;36(3):353-362.

80. Espie CA, Kyle SD. Therapy in Sleep Medicine: cognitive and behavioural psychological therapies for chronic insomnia. Barkoukis TJ, Matheson JK, Ferber R, Dohramji K, editors. Oxford: Elsevier Health Sciences. 2012;161-171.
ChronoPhysiology and Therapy

\section{Publish your work in this journal}

ChronoPhysiology and Therapy is an international, peer-reviewed, open access journal focusing on research into the cyclic variations and rhythmicity in physiological processes in the body and the research and development and optimal timing of administration of therapeutic targets to achieve improved outcomes and quality of life for the patient. The

\section{Dovepress}

manuscript management system is completely online and includes a very quick and fair peer-review system. Visit http://www.dovepress.com/ testimonials.php to read real quotes from published authors.

Submit your manuscript here: http://www.dovepress.com/chronophysiology-and-therapy-journal 